\title{
Efficacy of Intravitreal Bevacizumab in Chronic Central Serous Chorioretinopathy in Terms of Mean Reduction of Central Macular Thickness in Pakistani Patients
}

\author{
Huzaifa Akhlaq1', Muhammad Nawaz Malik2, Ejaz Ahmad Javed ${ }^{3}$, Iftikhar Ahmad ${ }^{4}$, Shahzada Najam Ul Hassan ${ }^{5}$
}

1 Senior Registrar, Department of Ophthalmology, Allied Hospital, Faisalabad Pakistan

Study designing, Data collection, Results and discussion writing

2 Professor E Head, Department of Ophthalmology, Faisalabad Medical University/Allied Hospital, Faisalabad Pakistan

2 Critical review, Proof reading

3 Associate Professor, Department of Ophthalmology, Faisalabad Medical University/Allied Hospital, Faisalabad Pakistan Statistical analysis

4 Assistant Professor, Department of Ophthalmology, Faisalabad Medical University/DHQ Hospital, Faisalabad Pakistan

4 Help in designing the study, Tabulation of results

5 Assistant Professor, Department of Ophthalmology, Faisalabad Medical University/Allied Hospital, Faisalabad Pakistan Literature review

\author{
Submitted for Publication: 07-09-2019 \\ Accepted for Publication 21-12-2020 \\ CORRESPONDING AUTHOR \\ Dr. Huzaifa Akhlaq \\ Senior Registrar, Department of \\ Ophthalmology, Allied Hospital, Faisalabad \\ Pakistan \\ Email: dr.huzaifa.akhlaq@gmail.com
}

\section{ABSTRACT}

Background: Central serous chorioretinopathy (CSCR) is an idiopathic condition described by serous neurosensory retinal detachment at the posterior pole, usually associated with serous retinal pigment epithelial detachment (PED). CSCR patients as a rule have good visual prognosis. In most of the patients, CSCR is self-limiting and patients regain the last visual acuity. However, some patients may have visual disability due to permanent RPE and photoreceptor damage. Objective: The objective of the study is to determine the effect of intravitreal Bevacizumab in cases of chronic central serous chorioretinopathy in terms of mean change in central macular thickness. Study Design: Interventional experimental study. Settings: The study was conducted in Department of Ophthalmology, Allied Hospital, Faisalabad Pakistan. Duration: 2 years from January 2017 to December 2018. Methodology: An aggregate of 95 cases were recruited for the study. Study included both male and female patients with diagnosis of chronic CSCR (at least 6month duration). Best corrected visual acuity, Intra Ocular Pressure on Goldman applanation Tonometer and Optical coherence tomography on Optovue were recorded of both eye of all patients. Before starting the treatment, informed consent was signed by every patient and they were called on the day of injection. Intravitreal off label Bevacizumab $(1.25 / 0.05 \mathrm{ml})$ was given through pars plana in operation theater under aseptic conditions. After injection topical antibiotic drops were given to patients for one week and patients called for follow up after one week, one month, 2 month and 3 months. Same parameters BCVA, IOP, Dilated fundus examine and OCT was recorded on every visit. The final outcome i.e., change in central macular thickness was recorded. Results: In this study, out of 95 cases, $53.68 \%(n=51)$ were between $30-45$ years of age while $46.32 \%(n=44)$ were between $46-60$ years of age, mean \pm SD was calculated as $46.83 \pm 7.82$ years, $74.74 \%(n=71)$ were male and $25.26 \%(n=24)$ were females. Pre-treatment mean central macular thickness was recorded as $344.77 \pm 14.61 \mu$ which reduced to $154.57 \pm 16.57 \mu$, mean difference was recorded as $190.2 \pm 21.46 \mu, \mathrm{p}$ value was 0.0001 showing a significant difference. Conclusion: We concluded that intravitreal Bevacizumab significantly reduced central macular thickness in cases of chronic central serous chorioretinopathy.

Keywords: Central serous chorioretinopathy, Intravitreal Bevacizumab, Mean change in central macular thickness, Anti-VEGF.

How to Cite: Akhlaq H, Malik MN, Javed EA, Ahmad I, Hassan SN. Efficacy of Intravitreal Bevacizumab in Chronic Central Serous Chorioretinopathy in Terms of Mean Reduction of Central Macular Thickness in Pakistani Patients. APMC 2020;14(4):349-52. DOI: 10.29054/ APMC/2020.576

\section{INTRODUCTION}

Central serous chorioretinopathy (CSC) is an idiopathic retinopathy, defined as serous detachment of the neurosensory retina at the macula. ${ }^{1}$ The problem is usually self-limiting, though a few patients are left with lasting visual impairment resulting from permanent damage to RPE and photoreceptors. ${ }^{2}$ Almost 5\% of patients experiences poor visual outcome due to permanent damage to RPE \& Photoreceptor. Normally CSCR affects young males and mostly one eye is involved $^{3}$ but there are cases in which both eyes of the same patient are affected. But on OCT, normal eye has also changes at the level of RPE in form of small serious PED and retino-choroidal thickening.

Late indocyanine green angiography in patients with CSCR has displayed confirmation of choroidal lobular ischemia and choroidal venous blockage, and moreover it revealed various zones of choroidal vascular hyperpermeability. ${ }^{4}$ 
Bevacizumab (Inj. Avastin by Roche, Basel, Switzerland), a monoclonal anti-body which blocks Vascular Endothelial Growth Factor (VEGF), is used in diabetic macular edema and CNV's. ${ }^{5}$ There have been a couple of off-label clinical studies of intravitreal Bevacizumab use in CSCR. 6,7 Most of the studies concluded positive results with improved visual outcome and decreased sub-retinal fluid.

Lim SJ and others ${ }^{8}$ assessed the efficacy of intravitreal injection of Bevacizumab for treatment of chronic central serous chorioretinopathy and recorded that mean macular thickness decreased from $331.5+93.4 \mu$ to $164+34 \mu$ at 3 months $(\mathrm{P}=0.043)$ demonstrating a mean reduction of $167.5+93.06 \mu$. Another study ${ }^{9}$ recorded a change in CMT that was not clinically significant $(-8.37 \mu \mathrm{m}, 95 \%$ CI-97.26 to $80.52, \mathrm{p}=0.85$ ).

Rationale: The results of previous studies regarding the efficacy of Intravitreal Bevacizumab have high variations. The rationale of this study is to investigate the efficacy of intravitreal use of Bevacizumab in chronic central serous chorioretinopathy in local patients of Pakistan. The results of this study will be useful for the Ophthalmologists and the patients with chronic central serous chorioretinopathy.

The objective of the study is to determine the effect of intravitreal Bevacizumab in cases of chronic central serous chorioretinopathy in terms of mean change in central macular thickness.

\section{METHODOLOGY}

Study Design: Interventional experimental Study.

Settings: Department of Ophthalmology, Allied Hospital, Faisalabad Medical University, Faisalabad Pakistan.

Duration: Two years from January 01, 2017 to December 31, 2018.

Sample Technique: Non-probability consecutive sampling.

Sample Size: The sample size of 95 cases, calculated with $95 \%$ confidence level, $\mathrm{d}=1$ and taking expected change in CMT at 3 months after intravitreal Bevacizumab in cases with Chronic central serous chorioretinopathy i.e., $167.5+93.06 \mu 9$.

Inclusion Criteria:

- Age: 30-60 years.

- Both gender: Male / Female.

- All diagnosed cases of Chronic Central serous chorioretinopathy (at least 6 months duration).

Exclusion Criteria:

- Sub-retinal Choroidal neovascular membrane.

- Prior treatment with laser photocoagulation, Transpupillary thermotherapy or photodynamic therapy.

- History of thromboembolic events including stroke, transient ischemic attacks and myocardial infarction.
- History of previous treatment with intravitreal antiVEGF.

- Intraocular pressure more than $21 \mathrm{mmHg}$.

- History of retinal detachment, intraocular inflammation.

Data Collection Procedure: A total of 95 cases fulfilling the inclusion/exclusion criteria were enrolled from Department of Ophthalmology, Allied Hospital, Faisalabad. The study was conducted after the approval of Ethical Research Committee (ERC) of the University. An informed consent of all the patients was obtained to include them in the study. Socio-demographic profile like name, age, gender and history of current disease with respect to symptoms, severity and duration was taken. At baseline and follow-up visits, examination included detailed anterior segment examination with slit lamp, visual acuity with Snellen's chart, intraocular pressure measurement with Goldman's applanation tonometer, dilated fundus examination and Optical Coherence Tomography of macula on Optovue.

Outcome measure was change in central macular thickness. The intravitreal injection of off label Bevacizumab was performed in a standard protocol in the operation theatre under complete aseptic conditions. Post injection follow-up included repeated clinical examination. Follow-up visits were scheduled to next day, 1 week, then monthly till the end of follow-up at 3 months. The final outcome i.e., change in central macular thickness was recorded. All the information was recorded on a pre-designed proforma.

Data Analysis Procedure: The collected data was entered into SPSS version 14 and analyzed descriptively. Mean \pm standard deviation was calculated for quantitative variables i.e., age, and central macular thickness (baseline, post-op, change). Frequency and percentage were calculated for gender of the patients. Un-paired sample t-test was applied for pre \& post treatment change in CMT, $p$ value of $\leq 0.05$ was taken as significant. The data was stratified for age, and gender of the cases to control the effect modifiers. Post stratification un-paired $t$-test was used by taking $p$ value of $\leq 0.05$ as significant.

\section{RESULTS}

A total of 95 cases fulfilling the inclusion/exclusion criteria were enrolled to determine the effect of intravitreal Bevacizumab in cases of chronic central serous chorioretinopathy in terms of mean change in central macular thickness.

Age distribution of the patients showed that $53.68 \%(n=51)$ were between $30-45$ years of age while $46.32 \%(n=44)$ were between $46-60$ years of age, mean $( \pm$ SD) age was calculated as $46.83 \pm 7.82$ years. (Table 1 ) 
Table 1: Age distribution $(\mathrm{n}=95)$

\begin{tabular}{|c|c|c|}
\hline Age (in years) & No. of patients & $\%$ \\
\hline $\mathbf{3 0 - 4 5}$ & 51 & 53.68 \\
\hline $\mathbf{4 6 - 6 0}$ & 44 & 46.32 \\
\hline Total & 95 & 100 \\
\hline Mean \pm SD & $46.83 \pm 7.82$ \\
\hline
\end{tabular}

Gender distribution showed that $74.74 \%(\mathrm{n}=71)$ were male and $25.26 \%(n=24)$ were females. (Table 2 )

Table 2: Gender distribution $(\mathrm{n}=95)$

\begin{tabular}{|c|c|c|}
\hline Gender & No. of patients & $\%$ \\
\hline Male & 71 & 74.74 \\
\hline Female & 24 & 25.26 \\
\hline Total & 95 & 100 \\
\hline
\end{tabular}

Pre-treatment mean central macular thickness was recorded as $344.77 \pm 14.61 \mu \mathrm{m}$ which reduced to post treatment $154.57 \pm 16.57 \mu \mathrm{m}$, mean difference was recorded as $190.2 \pm 21.46 \mu \mathrm{m}, \mathrm{p}$ value was 0.0001 showing a clinically significant difference. (Table 3 )

Table 3: Mean central macular thickness(n=95)

\begin{tabular}{|c|c|c|}
\hline Central macular thickness $(\boldsymbol{\mu})$ & Mean & \pm SD \\
\hline Pre-treatment & 344.77 & 14.61 \\
\hline Post-treatment & 154.57 & 16.57 \\
\hline Mean difference & 190.2 & 21.46 \\
\hline
\end{tabular}

P value: 0.0001

The data stratified for age showed 30-45 year of age had pre-treatment mean central macular thickness of $343.86 \pm 14.89 \mu \mathrm{m}$ which reduced to $156.14 \pm 16.85 \mu \mathrm{m}$, mean difference was recorded as $187.72 \pm 1.96 \mu \mathrm{m}, \mathrm{p}$ value was 0.0001 showing a significant difference. Patients between 45-60 year of age had pre-treatment mean central macular thickness of $345.82 \pm 14.37 \mu \mathrm{m}$ which reduced to $152.75 \pm 16.24 \mu \mathrm{m}$, mean difference was recorded as $193.70 \pm 1.87 \mu \mathrm{m}, \mathrm{p}$ value was 0.0001 showing a significant difference. (Table 4)

Table 4: Stratification for mean central macular thickness with regards to age

\begin{tabular}{|c|c|c|c|c|}
\hline \multirow{2}{*}{ Age group } & \multicolumn{3}{|c|}{ Change in CMT } & \multirow{2}{*}{ P value } \\
\cline { 2 - 4 } & $\mathbf{N}$ & Mean & $\mathbf{\pm S D}$ & \\
\hline $\mathbf{3 0 - 4 5}$ & 51 & 187.73 & 20.90 & \multirow{2}{*}{0.22} \\
\hline $\mathbf{4 6 - 6 0}$ & 44 & 193.07 & 21.99 & \\
\hline
\end{tabular}

The data stratified for gender showed that male cases had pre-treatment mean central macular thickness of $343.25 \pm 14.25 \mu \mathrm{m}$ which reduced to $154.63 \pm 18.27 \mu \mathrm{m}$, mean difference was recorded as $188.62+4.02 \mu \mathrm{m}, \mathrm{p}$ value was 0.0001 showing a significant difference. Female patients had pre-treatment mean central macular thickness of $349.25 \pm 15.05 \mu \mathrm{m}$ which reduced to $154.38 \pm 10.27 \mu \mathrm{m}$, mean difference was recorded as $194.87 \pm 4.78 \mu \mathrm{m}$, $\mathrm{p}$ value was 0.0001 showed a significant difference. (Table 5)

There was no significant difference of change in central macular thickness among male and females. (Table 5)

Table 5: Stratification for mean central macular thickness with regards to gender

\begin{tabular}{|c|c|c|c|c|}
\hline \multirow{2}{*}{ Gender } & \multicolumn{3}{|c|}{ Change in CMT } & \multirow{2}{*}{ P value } \\
\cline { 2 - 4 } & $\mathbf{N}$ & Mean & \pm SD & \\
\hline Male & 71 & 188.62 & 22.58 & \multirow{2}{*}{0.218} \\
\hline Female & 24 & 194.87 & 17.33 & \\
\hline
\end{tabular}

\section{DISCUSSION}

CSCR is a benign self-limited condition characterized by idiopathic serous detachment of the neurosensory retina. A number of hypotheses have been proposed regarding the pathophysiology of CSCR. Dysfunction of the RPE with reversal of liquid transport may play a role in the development of serous retinal detachment. Studies based on ICGA findings in patients with CSCR have demonstrated evidence of choroidal lobular ischemia, choroidal venous congestion and multiple areas of vascular hyperpermeability. Choroidal hyperpermeability may cause RPE decompensation and damage with subsequent leakage of fluid into the subretinal space. RPE damage may also occur by shedding of outer photoreceptor segments with a primarily intact blood-retinal barrier and result in accumulation of fluid in the subretinal space. ${ }^{10}$

There is no established treatment for CSCR. The high spontaneous remission rate favors conservative management and lifestyle counseling as the first-line of therapy. Spontaneous resolution of detachment without any intervention is expected in approximately $90 \%$ of cases. ${ }^{11}$ If the detachment persists for more than six months, intervention may be considered necessary. In some cases, earlier intervention may be necessary due to high occupational demands for binocular vision. Although there is no strong evidence for early treatment of CSCR, many retina specialists suggest that Acetazolamide, laser photocoagulation, PDT or intravitreal anti-VEGF injections be used. There are some studies supporting the benefit of early treatment of CSCR. These studies propose that the potential advantage of early resolution may be mediated by a lower rate of RPE degeneration in treated eyes. However, there are complications associated with focal thermal laser photocoagulation and PDT.12 These include scotoma formation, loss of contrast sensitivity, foveal damage, RPE damage, choroidal ischemia and choroidal 
neovascularization. Therefore, these modalities should be used with caution in such eyes with a high potential for spontaneous recovery.

The vascular endothelial growth factor (VEGF) is a wellknown and potent inducer of vascular permeability. Bevacizumab is a full-length antibody that binds all isoforms of VEGF. There are many reports on the efficacy and safety of intravitreal Bevacizumab in retinal disorders. Nevertheless, the role of anti-VEGF agents for treatment of CSCR is not well-known. In some studies, the possible benefits of an anti-VEGF agent in CSCR were proposed on the basis of choroidal ischemia and hyperpermeability as pathogenetic mechanisms of CSCR. Choroidal ischemia may cause an increase in the concentration of VEGF, and anti-VEGF agents may reduce choroidal hyperpermeability by blocking VEGF activity. Although several reports have supported the efficacy of IVB injection in CSCR, there are no studies demonstrating increased levels of VEGF in CSCR. Even in a study on VEGF levels in the aqueous humor, the difference between the concentration of VEGF in the aqueous humor of CSCR versus controls was not significant.

During recent study 95 cases were selected from Department Ophthalmology Allied Hospital Faisalabad. $74.74 \%(\mathrm{~N}=71)$ were male and $25.26 \%(\mathrm{n}=24)$ were female. Their ages ranges between 30- 60 years. Pre- treatment mean central macular thickness was recorded $344.77 \pm$ $14.6 \mathrm{um}$ which reduced to $154.57 \pm 16.57 \mathrm{um}$. Mean difference was recorded as $190.2 \pm 21.46 \mathrm{um}, \mathrm{p}$ value shows a significance difference.

The safety profile of IVB appears favorable due to a low rate of ocular and systemic adverse events; however, it is not a procedure with zero risks and serious complications such as cataracts, retinal breaks, endophthalmitis and even death due to thromboembolic events may occur. Patients with CSCR are relatively young and have a high life expectancy. The possibility of adverse events with IVB injection should be considered seriously in these patients. None of the patients in the present study experienced a significant adverse event associated with IVB injection.

In summary, we found significant decrease in of central macular thickness in patients of chronic central serous chorioretinopathy, who have received intravitreal Bevacizumab.

\section{CONCLUSION}

We concluded that intravitreal Bevacizumab significantly reduces central macular thickness in cases with chronic central serous chorioretinopathy.

\section{LIMITATIONS}

Participants of study belongs to Faisalabad city and periphery of Faisalabad. Large multi centre trial is needed to reach a conclusion and to standardize the treatment.

\section{SUGGESTIONS / RECOMMENDATIONS}

This study is conducted in cases of chronic CSCR. Further multi-Centre study is recommended in Acute CSCR as well.

\section{CONFLICT OF INTEREST / DISCLOSURE}

No conflict of interest.

\section{ACKNOWLEDGEMENTS}

Authors acknowledge seniors and colleagues of the Department for their continuous support and guidance

\section{REFERENCES}

1. Li XJ, Zhang JS. Intravitreal bevacizumab injection for chronic central serous chorioretinopathy. Chin Med J (Engl). 2010;123(15):2145-7.

2. Jamil AZ, Rahman F, Iqbal K, Ansari HM, Iqbal W, Mirza KA. Intravitreal Bevacizumab in Central Serous Chorioretinopathy. JCPSP. 2012;22(6):363-6.

3. Hussain, Khan MT, Rehman H, Ziauddin. Role of Intravitreal Bevacizumab in the Treatment of Acute Central Serous Chorioretinopathy. Ophthalmology. 2013;11:30-3.

4. Lim SJ, Roh MI, Kwon OW. Intravitreal bevacizumab injection for central serous chorioretinopathy. Retina. 2010;30:100-6.

5. Chung YR, Seo EJ, Lew HM, Lee KH. Lack of positive effect of intravitreal bevacizumab in central serous chorioretinopathy: meta-analysis and review. Eye (Lond). 2013;27(12):1339-46.

6. Heiduschka P, Fietz H, Hofmeister S, Schultheiss S, Mack AF, Peters S. Penetration of bevacizumab through the retina after intravitreal injection in the monkey. Invest Ophthalmol Vis Sci. 2007;48(6):2814-23.

7. Scott IU, Edwards AR, Beck RW, Bressler NM, Chan CK, Elman MJ. A phase II randomized clinical trial of intravitreal bevacizumab for diabetic macular edema. Ophthalmology. 2007;114(10):1860-67.

8. Soheilian M, Ramezani A, Obudi A, Bijanzadeh B, Salehipour M, Yaseri M. Randomized trial of intravitreal bevacizumab alone or combined with triamcinolone versus macular photocoagulation in diabetic macular edema. Ophthalmology. 2009;116 (6):1142-50.

9. Sharma S, Johnson D, Abouammoh M, Hollands S, Brissette A. Rate of serious adverse effects in a series of bevacizumab and ranibizumab injections. Can J Ophthalmol. 2012;47 (3):275-79.

10. Semeraro F, Romano MR, Danzi P, Morescalchi F, Costagliola C. Intravitreal bevacizumab versus low-fluence photodynamic therapy for treatment of chronic central serous chorioretinopathy. Jpn J Ophthalmol. 2012;56(6):608-12.

11. Lee JY, Chae JB, Yang SJ, Kim JG, Yoon YH. Intravitreal bevacizumab versus the conventional protocol of photodynamic therapy for treatment of chronic central serous chorioretinopathy. Acta Ophthalmol. 2011;89(3):293-4.

12. Koss MJ, Beger I, Koch FH. Subthreshold diode laser micropulse photocoagulation versus intravitreal injections of bevacizumab in the treatment of central serous chorioretinopathy. Eye (Lond). 2012;26(2):307-14. 